\title{
A prospective study on neoadjuvant chemoradiotherapy plus anti-EGFR monoclonal antibody followed by surgery for locally advanced cervical cancer
}

This article was published in the following Dove Press journal: OncoTargets and Therapy

Heming $L^{1,2}$

Yuying $\mathrm{Wu}^{3}$

Xu Liu ${ }^{2}$

Hailan Jiang ${ }^{2}$

Qiang Pang ${ }^{2}$

Luxing Peng ${ }^{2}$

Jinjian Cheng ${ }^{2}$

Shan Deng ${ }^{2}$

Junzhao $\mathrm{Gu}^{2}$

Renfeng Zhao ${ }^{3}$

Xiaoxia $\mathrm{Hu}^{3}$

Changyi Chen ${ }^{3}$

Jinming $\mathrm{Yu}^{\prime}$

'Department of Radiation Oncology, Shandong Cancer Hospital Affiliated to Shandong University, Jinan, China; ${ }^{2}$ Department of Radiation Oncology, People's Hospital of Guangxi Zhuang Autonomous Region, Nanning,

China; ${ }^{3}$ Department of Gynecology, People's Hospital of Guangxi Zhuang Autonomous Region, Nanning, China

Correspondence: Jinming Yu Department of Radiation Oncology, Shandong Cancer Hospital Affiliated to Shandong University, No 440 Jiyan Road, Jinan 250 I I7, China

Tel +86053l87984777

Email sdyujinming@।63.com
Background: To investigate the efficacy and safety of neoadjuvant chemoradiotherapy plus anti-epidermal growth factor receptor monoclonal antibody followed by surgery for locally advanced cervical cancer (LACC).

Patients and methods: Patients with histologically proven LACC were enrolled into this prospective study. All patients received intensity-modulated radiation therapy with conventional fractionation. Weekly cisplatin or nedaplatin was administered concurrently with intensitymodulated radiation therapy. Nimotuzumab, a humanized anti-epidermal growth factor receptor monoclonal antibody, was given at a dose of $200 \mathrm{mg}$ per week for 6 cycles. Approximately 1 month after the completion of neoadjuvant treatment, the patients were assessed for clinical tumor response and operability based on MRI and gynecological examination. For those who were considered to be candidates for surgery, radical hysterectomy, and pelvic lymph node dissection were performed 5-6 weeks after the completion of neoadjuvant therapy.

Results: Twenty-eight patients were enrolled. Clinical complete response and partial response were found in $8(28.5 \%)$ and $20(71.5 \%)$ patients, respectively. Four patients were not eligible for surgery and 2 patients refused surgery although they were assessed as surgical candidates. They were not included in this analysis. Radical hysterectomy and pelvic lymph node dissection were performed for the remaining 22 patients. Among them, 8 (36.4\%) had complete pathology response, $9(40.9 \%)$ presented with persistent atypical cells or cervical intraepithelial neoplasia, and $5(22.7 \%)$ presented with macroscopic and/or microscopic residual disease, according to the pathological evaluation. Median follow-up time was 22 months (range, 5-39 months). The 2-year locoregional control rate, progression-free survival rate, distant metastasis-free survival rate, and overall survival rate were $95.0 \%, 85.2 \%, 84.0 \%$, and $90.0 \%$, respectively. Acute toxicities were mild in general and easily manageable. Chronic toxicities were mainly limited to grade 1 . No severe late toxicities were observed.

Conclusion: Concurrent chemoradiotherapy plus nimotuzumab followed by surgery is highly effective and safe in LACC. Further studies are warranted to confirm the findings.

Keywords: locally advanced cervical cancer, neoadjuvant chemotherapy, intensity-modulated radiotherapy, anti-EGFR monoclonal antibody, radical surgery, hysterectomy

\section{Introduction}

Cervical cancer is a serious threat to the health of female population worldwide, particularly in developing countries where over $80 \%$ of women with cervical cancer are diagnosed at advanced stages. ${ }^{1}$ For patients with locally advanced cervical cancer 
(LACC), concurrent chemoradiotherapy (CCRT) as a standard treatment approach, has achieved better survival, local control and distant control when compared with radiation therapy (RT) alone, as shown in several studies. ${ }^{2-4}$

Over the last decade, neoadjuvant chemotherapy followed by surgery has also been widely explored. However, the results are inconsistent. ${ }^{5-8}$ Experiences from cancers in other sites such as head-and-neck cancer and rectal cancer suggest that additional RT to neoadjuvant chemotherapy prior to surgery may have a role in LACC. Ferrandina et al conducted a series of studies and preliminarily confirmed that CCRT followed by surgery was both effective and tolerable. ${ }^{9,10}$

The expression of epidermal growth factor receptor (EGFR) gene is closely associated with tumor cell growth, proliferation, invasion, metastasis, and apoptosis. ${ }^{11}$ Previous studies have indicated that EGFR can be an appropriate target in cervical cancer as its overexpression ranges from $6 \%$ to $90 \%$, and some studies also found poor prognosis associated with the presence of EGFR. ${ }^{12}$ Blocking EGFR pathway with anti-EGFR antibody may inhibit tumor cell proliferation, differentiation, tumor angiogenesis, and promote treatment response of chemotherapy and radiation. ${ }^{11}$ Nimotuzumab, as a humanized monoclonal antibody targeting EGFR, has been tested in the setting of recurrent, persistent, or metastatic cervical cancer, when combined with chemotherapy. ${ }^{13}$ It was well tolerated and may have an important role in the treatment of LACC. However, to the best of our knowledge, there is no study focusing on the role of nimotuzumab in neoadjuvant setting. The current study was designed to investigate the efficacy and safety of CCRT plus nimotuzumab followed by surgery for LACC.

\section{Patients and methods Eligibility criteria}

The inclusion criteria of this study were as follows: Histologically proven squamous cell carcinoma or adenocarcinoma; age: 18-75 years old; Eastern Cooperative Oncology Group (ECOG) performance status $<2$; International Federation of Gynecology and Obstetrics stage IB2-IIIB; normal bone marrow function. The exclusion criteria included pregnant or lactating women, patients with other malignancies, patients who received upfront radiotherapy or chemotherapy, presence of uncontrolled life-threatening illness, or allergy to platinum or monoclonal antibody. Informed study-specific consent forms were signed by the patients. The study was registered with ClinicalTrials.gov (No NCT01938105) and approved by the Institutional Review Board of the People's Hospital of Guangxi Zhuang Autonomous Region.

Pretreatment work up was required for all patients, including review of medical history, clinical examination, gynecological examination, chest $\mathrm{CT}$, abdominal ultrasound, pelvic MRI, and emission CT bone scan as indicated, to determine the extent of the disease. In addition, all patients were evaluated for complete blood cell count, and hepatic and renal function before entry into the study.

\section{Intensity-modulated radiation therapy (IMRT)}

Thirty minutes before CT simulation and each subsequent treatment, patients were asked to empty their rectum and bladder, and then drink $500 \mathrm{~mL}$ of water and hold urine. Oral contrast medium was also administered at this time. During the simulation, patients were required to lie down on a wide-bore CT simulator couch (Somatom Sensation Open, Siemens Medical Solutions, Erlangen, Germany) in a supine position. Tailored thorocoabdominal thermoplastic masks were designed to cover the lower chest, abdomen, and pelvis. Intravenous contrast-enhanced CT using $4 \mathrm{~mm}$ slice from the upper border of the T12 vertebral to $3 \mathrm{~cm}$ below the ischial tuberosity was performed for planning. The CT data were imported to the Pinnacle ${ }^{3}$ system (version 9.2, Philips Radiation Oncology Systems, Fitchburg, WI, USA).

Gross tumor volume (GTV) was defined as any primary disease and involved lymphadenopathy, determined by the imaging and clinical findings. There were 3 clinical tumor volumes (CTV1, CTV2, and CTV3). CTV1 included GTV, uterus, and cervix. CTV2 included parametrial and paravaginal tissues, ovaries, and vagina according to the involvement (ie, upper half if no vagina involvement or only slight involvement; upper two-thirds if upper vagina involvement; or entire if more vagina involvement). CTV3 included common iliac, internal iliac, external iliac, and presacral lymph nodes. The margins between PTV1 and CTV1, between PTV2 and CTV2, and between PTV3 and CTV3 were 10, 7, and $7 \mathrm{~mm}$, respectively. The contoured critical structures included the rectum, small bowel, bladder, femoral heads, and pelvic bone marrow.

The plans were designed and optimized using the Pinnacle inverse planning system. The prescribed radiation doses delivered to PTV1, PTV2, and PTV3 were 50-54 Gy at 1.95-2.12 Gy per fraction, $45-48.6$ Gy at $1.80-1.86$ Gy per fraction, and 45-48.6 Gy at 1.70-1.80 Gy per fraction, respectively, in 25-27 fractions. IMRT was delivered via 5 or 7 fixed-gantry angles with an Elekta Synergy Linear Accelerator (Elekta Ltd., Stockholm, Sweden) with step-and-shoot treatment techniques. It should be noted that for patients whose treatment fractions were scheduled to be $<5$ during the first week or the last week, we usually provided an additional treatment at the first weekend or the previous weekend of the last week. 
For example, if a patient started IMRT on Tuesday at the first week, then an additional treatment was delivered at the first weekend. Likewise, if she was initially assigned to finish her treatment on the days except for Friday, an additional treatment would be offered during the previous weekend. Kilovoltage Cone beam CT was performed 3-5 times weekly prior to IMRT, to facilitate patient setup.

\section{Anti-EGFR and concurrent chemotherapy}

Weekly nimotuzumab $(200 \mathrm{mg})$ diluted in normal saline to a total volume of $250 \mathrm{~mL}$ was administered by intravenous infusion over $60 \mathrm{~min}$ for 6 cycles, starting from the first week of IMRT. Weekly cisplatin $\left(40 \mathrm{mg} / \mathrm{m}^{2}\right)$ or nedaplatin $\left(30 \mathrm{mg} / \mathrm{m}^{2}\right)$ was administered concurrently with IMRT. Antiemetic medication was given routinely during chemotherapy administration. Patients were expected to receive 5-6 cycles of concurrent chemotherapy.

\section{Surgery and pathological workup}

Both gynecological examination and MRI scan were initiated to assess clinical response upon the completion of neoadjuvant treatment, according to the Response Evaluation Criteria in Solid Tumors (RECIST). Approximately 1 month after the completion of neoadjuvant treatment, patients were required to receive an assessment for operability consulted by senior gynecological physicians. In general, operability was defined if a patient had no palpable/visible disease in parametrium and ligamentum sacrouterinum, and no distal parametrial involvement. For those considered as candidates for surgery, radical hysterectomy and pelvic lymphadenectomy were performed 5-6 weeks after IMRT. Pathological response to neoadjuvant treatment was evaluated based on the histopathological examination of resected specimen. Complete response (CR) was defined as complete disappearance of all macroscopic and microscopic diseases and mainly showing inflammatory cell infiltration. Partial response (PR) was defined as presence of persistent atypical cells or cervical intraepithelial neoplasia. And residual carcinoma (RC) was defined as macroscopic and/or microscopic residual disease. Immunohistochemical staining was used for the pretreatment biopsied tumor samples to determined EGFR expression. Low-to-moderate and high EGFR expressions were defined as $26 \%-90 \%$, and $>90 \%$ positive tumor cells, respectively, as described by Basavaraj et $\mathrm{al}^{14}$ (Figure 1).

\section{Toxicity assessment}

Toxicity assessment was performed according to Radiation Therapy Oncology Group criteria and Common Toxicity Criteria for Adverse Event v 3.0. Acute toxicities were

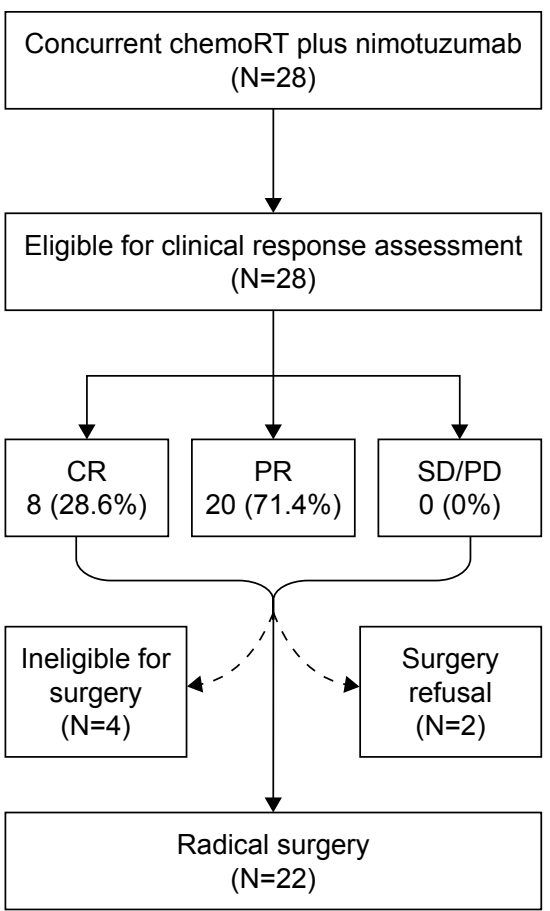

Figure I Study flowchart.

Abbreviations: CR, complete response; PD, progressive disease; PR, partial response; $\mathrm{RT}$, radiation therapy; $\mathrm{SD}$, stable disease.

evaluated weekly during the treatment until 3 months after the surgery, and late toxicities (occurring $>3$ months after the surgery) were evaluated once every 3 months for the first 2 years and every 6 months thereafter.

\section{Statistical consideration}

Progression-free survival (PFS) was defined as the time from patient recruitment to disease progression or death from any cause. Overall survival (OS) was defined as the time from patient recruitment to the date of death due to any causes or the date of last follow-up. Locoregional control rate (LRCR) was defined as the absence of either persistent or recurrent disease at the primary site or in the regional lymph nodes, and distant metastasis-free survival (DMFS) was defined as the time from the date of patient recruitment to the date of distant metastasis. Survival analyses were computed using the Kaplan-Meier method. Analyses were performed using SPSS 16.0 software (SPSS, Inc., Chicago, IL, USA).

\section{Results}

\section{Patient characteristics}

Between June 2013 and July 2016, a total of 28 patients diagnosed with LACC were enrolled into this study. The median age was 55 years (range, $31-75$ years old). Twenty-six patients $(92.9 \%)$ had squamous cell carcinoma and 2 patients (7.1\%) had adenocarcinoma. Stage distributions were as 
Table I Patient characteristics and treatment details

\begin{tabular}{|c|c|c|}
\hline Characteristics & $\begin{array}{l}\text { Number } \\
\text { of patients }\end{array}$ & $\%$ \\
\hline All cases & 28 & \\
\hline \multicolumn{3}{|l|}{ Age (years) } \\
\hline Range & $31-75$ & \\
\hline Median & 55 & \\
\hline \multicolumn{3}{|l|}{ Histology } \\
\hline Squamous cell carcinoma & 26 & 92.9 \\
\hline Adenocarcinoma & 2 & 7.1 \\
\hline \multicolumn{3}{|l|}{ FIGO stage } \\
\hline IB2 & 3 & 10.8 \\
\hline IIA & 5 & 17.9 \\
\hline IIB & 16 & 57.1 \\
\hline IIIA & 2 & 7.1 \\
\hline IIIB & 2 & 7.1 \\
\hline \multicolumn{3}{|c|}{ EGFR expression for all patients } \\
\hline Low-to-moderate & 8 & 28.6 \\
\hline High & 14 & 50.0 \\
\hline \multicolumn{3}{|c|}{ EGFR expression for surgical candidate } \\
\hline Low-to-moderate & 6 & 27.3 \\
\hline High & 12 & 54.5 \\
\hline \multicolumn{3}{|l|}{ IMRT duration (days) } \\
\hline Range & $32-49$ & \\
\hline Median & 34 & \\
\hline \multicolumn{3}{|c|}{ Interval between IMRT and surgery (days) ${ }^{\mathrm{a}}$} \\
\hline Range & $20-61$ & \\
\hline Median & 34 & \\
\hline \multicolumn{3}{|l|}{ Cycles of nimotuzumab } \\
\hline 5 cycles & 13 & 46.4 \\
\hline 6 cycles & 15 & 53.6 \\
\hline \multicolumn{3}{|l|}{ Cycles of chemotherapy } \\
\hline 5 cycles of cisplatin & 3 & 10.7 \\
\hline$\geq 5$ cycles of nedaplatin & 23 & 82.1 \\
\hline$<5$ cycles of nedaplatin & 2 & 7.2 \\
\hline
\end{tabular}

Note: aCalculated on 22 patients.

Abbreviations: EGFR, epidermal growth factor receptor; FIGO, Federation of Gynecology and Obstetrics; IMRT, intensity-modulated radiation therapy.

follows: stages IB2, 3 patients; stage IIA, 5 patients; stage IIB, 16 patients, stage IIIA, 2 patients, and stage IIIB, 2 patients. The median IMRT duration was 34 days (range, 32-49 days). The median time interval between IMRT completion and surgery was 34 days (range, 20-61 days) (Table 1).

\section{Treatment compliance}

Patient compliance to the planned treatment was well maintained. All 3 patients who were treated with concurrent cisplatin completed 5 cycles of chemotherapy as expected. Of the remaining 25 patients who were treated with concurrent nedaplatin, $23(92.0 \%)$ completed 5 or more cycles of chemotherapy. Thirteen patients $(46.4 \%)$ were administered with 5 cycles of nimotuzumab, and 15 patients (53.6\%) with 6 cycles, as shown in Table 1.

\section{Clinical and pathological response}

At the end of neoadjuvant treatment phase, clinical CR and PR were observed in 8 (28.6\%), 20 patients $(71.4 \%)$, respectively. No patients had stable disease or disease progression. Twenty-two patients underwent radical hysterectomy and pelvic lymphadenectomy and the other 6 did not due to patient refusal (2 patients) or inoperability (4 patients). They were treated with other approaches and not included in the survival analysis. Pathological CR, PR, and RC were documented in $8(36.4 \%), 9(40.9 \%)$, and $5(22.7 \%)$ patients, respectively.

\section{Issues related to surgery}

The median operating time was 183 minutes, ranging from 85 to 275 minutes. Median blood loss was $475 \mathrm{cc}$, ranging from 100 to $1,200 \mathrm{cc}$. Six patients $(27.2 \%)$ experienced blood loss exceeding $500 \mathrm{cc}$, and 4 patients (18.2\%) had blood transfusion.

\section{EGFR expression}

For all patients, low-to-moderate and high EGFR expressions were found in $8(28.6 \%)$ and 14 patients $(50.0 \%)$, respectively. For patients who underwent surgery, low-to-moderate and high EGFR expressions were found in $6(27.3 \%)$ and 12 patients (54.5\%), respectively, as shown in Table 1 .

\section{Toxicity}

In general, both acute and late toxicities were mild and acceptable. Grade 3 acute toxicities were observed in only 5 patients (17.9\%), manifested as bone marrow suppression. Other acute toxicities, including rash, vulvitis, and upper and lower gastrointestinal tract toxicity were limited to grade 2 or less. With respect to the late toxicities, no patients experienced severe treatment-related sequelae. Only 1 patient experienced grade 2 irradiation cystitis (Table 2).

\section{Survival outcome}

No patients experienced local failure. One patient had regional failure. Five patients developed distant metastasis: 2 patient had lung metastasis, 1 patient had bone metastasis, 1 patient had abdominal wall metastasis, 1 patient had multiple metastases to the lung, bone, and mediastinal and retroperitoneal lymph nodes. One patient died.

The median follow-up time was 22 months, ranging from 5 to 39 months. The 2-year OS, PFS, LRCR, and DMFS were $90.0 \%, 85.2 \%, 95.0 \%$, and $84.0 \%$, respectively (Figure 2). Stratification-based analysis was not possible because only a few events occurred. 
Table 2 Acute and late toxicities

\begin{tabular}{|c|c|c|c|c|}
\hline Toxicity type & Grade I (\%) & Grade 2 (\%) & Grade 3 (\%) & Grade 4 (\%) \\
\hline \multicolumn{5}{|l|}{ Acute toxicity ${ }^{\mathrm{a}}$} \\
\hline Bone marrow & $9(32.1)$ & $9(32.1)$ & $4(\mid 4.3)$ & - \\
\hline Rash & $2(7.1)$ & - & - & - \\
\hline Vulvitis & $10(35.7)$ & $18(64.3)$ & - & - \\
\hline Upper Gl tract & $16(57.1)$ & $4(14.3)$ & - & - \\
\hline Lower Gl tract & $19(67.9)$ & $8(28.6)$ & I (3.6) & - \\
\hline \multicolumn{5}{|l|}{ Late toxicity } \\
\hline Skin and subcutaneous tissue & $5(22.7)$ & - & - & - \\
\hline Leg edema and pain & $3(13.6)$ & - & - & - \\
\hline Irradiation enteritis & $2(7.1)$ & - & - & - \\
\hline Irradiation cystitis & $2(7.1)$ & I (3.6) & - & - \\
\hline Uronephrosis & $4(14.3)$ & - & - & - \\
\hline
\end{tabular}

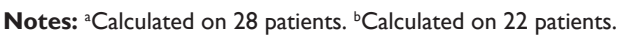

Abbreviation: $\mathrm{Gl}$, gastrointestinal.
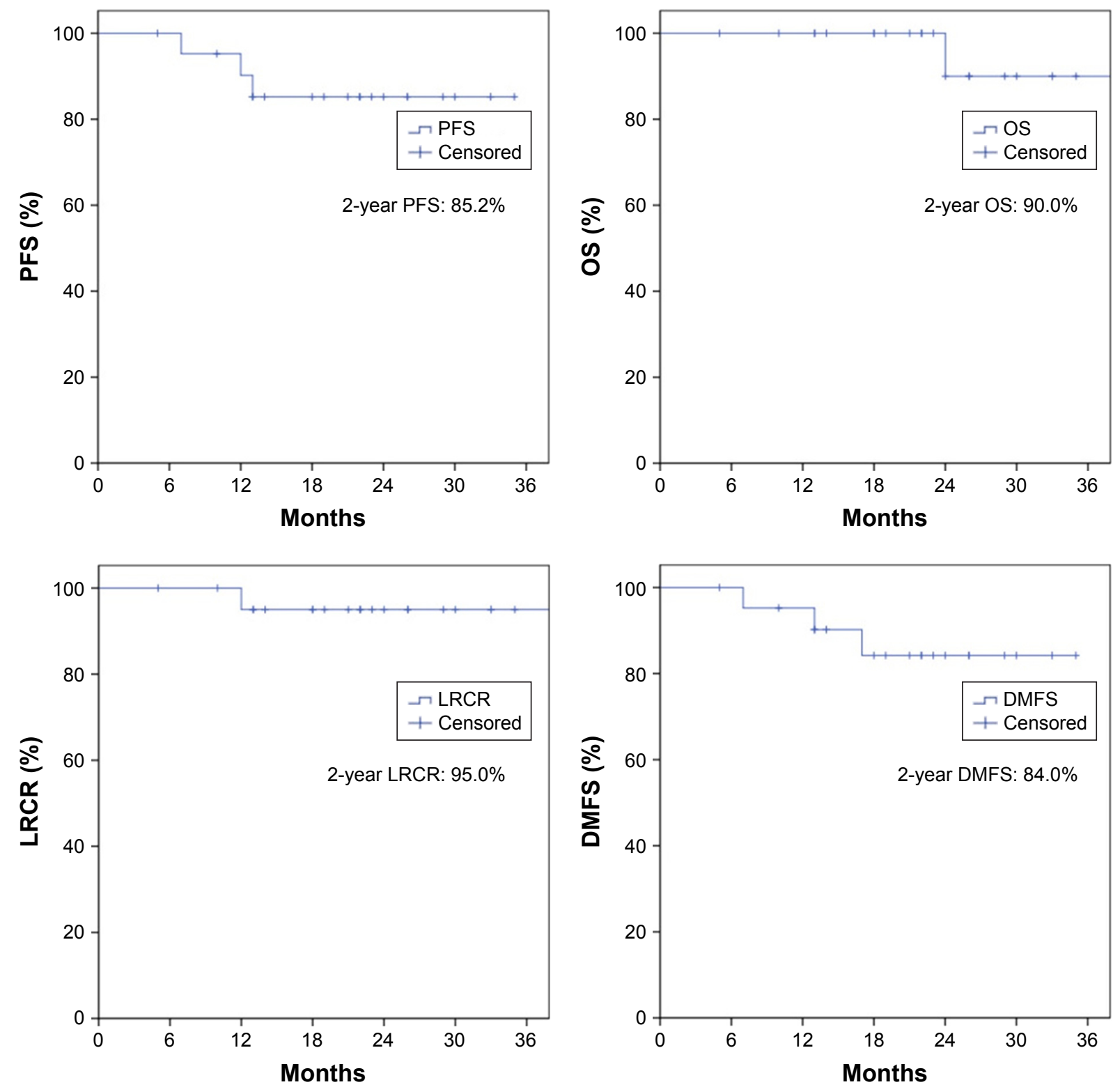

Figure 2 PFS, OS, LRCR, and DMFS in 22 patients treated with concurrent chemoradiotherapy plus nimotuzumab followed by radical surgery, estimated by the Kaplan-Meier method.

Abbreviations: DMFS, distant metastasis-free survival; LRCR, locoregional control rate; OS, overall survival; PFS, progression-free survival. 


\section{Discussion}

CCRT has long been considered the standard of care for LACC since the National Cancer Institute clinical alert was published in 1999. However, the treatment outcome is far from satisfactory, with about $70 \%$ of 5 -year OS. Besides locoregional relapse, distant metastasis remains the predominant cause of treatment failure. ${ }^{15,16}$ This situation leaves plenty of room to explore a more effective approach for further improvement. CCRT followed by radical surgery is one of the areas that researchers have being focused on in recent years.

In the present study, after the neoadjuvant treatment course, we observed CR and PR rates in $28.6 \%$ and $71.4 \%$, respectively. The overall response rate was $100.0 \%$. This higher objective response rate brought by neoadjuvant treatment is also reflected by other series although the $\mathrm{CR}$ rate varies largely. Ferrandina et $\mathrm{al}^{17}$ conducted a prospective study in which 161 patients diagnosed with LACC were treated with RT combined with cisplatin and 5-fluorouracil, followed by radical surgery, objective response was found in $96.8 \%$ of patients, $41.7 \%$ of whom achieved clinical CR. This was consistent with the recent findings reported by the same group in a large series, with $95.7 \%$ of objective response rate and $37.8 \%$ of clinical CR rate. ${ }^{10}$ More recently, a multicenter study enrolling 54 patient demonstrated a clinical CR rate of $31 \%$ after CCRT. ${ }^{18}$ Surprisingly, in another large study by Gong et al, a lower clinical CR rate $(2.43 \%)$ was reported despite $89.54 \%$ of clinical response rate. ${ }^{19} \mathrm{~A}$ possible reason for this lower CR rate may be attributed to the fact that those patients only received neoadjuvant chemotherapy and surgery without concurrent RT. In addition, it should be noted that the conventional imaging techniques exclusively depend on identifying morphologic criteria with a change in tumor dimension to evaluate the treatment response. However, these morphologic changes in gross tumor size significantly lag behind the biological and molecular changes that occur early in responders, thus findings of the conventional imaging are not consistently reliable to predict the treatment outcome..$^{18,20,21}$

For patients treated with CCRT followed by radical surgery, a critical issue that should not be ignored is the time interval between RT and surgery since it may be closely related with intra- and post-operative complications, and completeness of tissue resection as required. However, it has not been well defined so far. In the current study, radical surgery was performed at a median time of 34 days, ranging from 20 to 61 days. Ferrandina et al recommended a time interval of 5-6 weeks. In another study, the median time interval was
7 weeks (range, 4-14 weeks). ${ }^{17,22}$ Other researchers suggested that the radical surgery should be performed within 6-8 weeks. ${ }^{9,18,23}$ It seems that all the above recommendations are reasonable since the incidence of surgery-associated side effects was relatively low or the consequences were not severe. However, these recommendations rely largely on physician's experiences rather than research findings. Unlike other types of cancers such as cancers of rectum and breast treated in the same manner, cervical cancer has hardly been involved in the studies specifically focusing on the timing of postchemoradiotherapy surgery; thus, more efforts should be made to find out the optimal time interval between RT and radical surgery after a full consideration of both risks and benefits has been taken.

It has been found that pathological response is more closely associated with long-term treatment outcome than clinical response for patients with LACC. In the aforementioned study by Ferrandina et al, ${ }^{17} 44.1 \%$ of patients showed a pathological CR, $28.9 \%$ of patients showed a microscopic residual disease, and $27 \%$ of patients showed macroscopic residual disease. Regarding the prognosis, patients with absent/microscopic residual disease had a significantly longer disease-free survival (DFS) and OS than those with macroscopic disease (2-year DFS: $95 \%$ vs $58 \%, p=0.00001$; 2-year OS: $98 \%$ vs $62 \%, p=0.00001)$. The predictive value of pathological response on survival was demonstrated not only in stage IB2-II but also in stage III-IVA, indicating more effective modalities are needed to thoroughly eliminate the residual lesions and thus achieve a longer survival whenever possible. Comparing with the above study, patients in our series achieved slightly lower pathological CR (36.4\%). But the survival outcomes were also encouraging, with $90.0 \%$ of 2-year OS, $85.2 \%$ of PFS, $95.0 \%$ of LRCR, and $84.0 \%$ of DMFS. It is worth mentioning that the criteria for evaluating the pathological response in our study were not totally the same as those in other studies. For example, pathological PR in our study was defined as presence of persistent atypical cells or cervical intraepithelial neoplasia; whereas in Ferrandina's study, it was defined as persistence of only microscopic foci ( $\leq 3 \mathrm{~mm}$ maximum dimension) at any site level, and pathological CR was defined as the absence of any residual tumor. It is widely recognized that cervical intraepithelial neoplasia is a non-malignant lesion and usually curable. Therefore, some of our patients classified as having pathological PR may achieve pathological CR, according to the criteria in other studies., ${ }^{9,22}$ Given the higher pathological $\mathrm{CR}$ rate, improvement in survival would be anticipated if the follow-up period is long enough. Although local and regional 
tumor controls were excellent, distant metastasis was a major cause of treatment failure. Unfortunately, the association between pathological response and survival outcome was not established because only a fewer events were observed when the data were analyzed.

Treatment was well tolerated. All 3 patients who were administered concomitant cisplatin completed 5 cycles of chemotherapy as expected. Concomitant nedaplatin was given to the remainders, $92.0 \%$ of whom completed 5 or more cycles. All patients were administered $\geq 5$ cycles of nimotuzumab without treatment delay. The IMRT duration ranged from 32 to 49 days (median, 34 days). There was only 1 patient whose IMRT lasted $>40$ days because of hematological toxicity. In addition, no patients experienced intraoperative complications such as lymphocele, thrombophlebitis, wound dehiscence, or other surgery-related injuries. Hematological toxicity was the most common and severe acute toxicity. Other acute toxicities including upper and lower gastrointestinal tract side effect, vulvitis, and rash, were mild in general and easily manageable. Chronic toxicities were mainly limited to grade 2 or less. No severe late toxicities were observed.

Although cisplatin remains a cornerstone and standard agent for LACC, its side effects such as renal and gastrointestinal toxicities, poor treatment compliance, and additional preparation for hydration make it less attractive. As a second-generation platinum derivative, nedaplatin has been demonstrated to be equivalent to or have superior antineoplasic activity to cisplatin with less toxicity in cervical cancer. $^{24-26}$ The majority of our patient population was administered with weekly nedaplatin. Grade 3 acute toxicities were observed in only 4 patients (14.3\%), manifested as hematological toxicity. Patients also had a good compliance with nimotuzumab. As a single agent or in combination with chemotherapy or RT, nimotuzumab has been proven to be effective in a number of solid tumors, including head and neck cancer, rectum cancer, glioblastoma, and non-small-cell lung cancer. Notably, its side effects are less frequent and severe. ${ }^{27-29}$ In the present study, only 2 patients experienced skin rash, which was assigned as grade 1 toxicity and considered as certainly associated with the use of nimotuzumab. The toxicity profile can be explained by the fact that unlike other anti-EGFR antibody, nimotuzumab requires bivalent binding for stable attachment, resulting in a selective binding to EGFR-expressing cells. Comparing with tumor cells, the EGFR expression in normal tissues is much lower and their binding to nimotuzumab is transient, thus sparing normal tissues and subsequently avoiding severe toxicities. ${ }^{30}$
In cervical cancer, the predictive value of EGFR has not been clearly defined. EGFR overexpression has been found to be associated with poor prognosis in some studies but not in others. ${ }^{31,32}$ In the present study, EGFR overexpression was found in most subjects $(78.6 \%$ for all patients, and $81.8 \%$ for patients undergoing surgery). However, the correlation between EGFR and prognosis needs to be assessed.

\section{Conclusion}

The preliminary results of our study have shown that CCRT plus nimotuzumab followed by radical surgery is highly effective and safe in LACC. Longer follow-up is needed, and further randomized studies are warranted to confirm the findings.

\section{Acknowledgments}

This work was supported by the Wu Jieping Medical Foundation (No 2013-428-2081), Research and Development of Appropriate Medical Technology in Guangxi (S2017082), and Shandong Provincial Key Project of Translational Medicine (No ZR2015HZ004). This work was presented at the 53rd Annual Meeting of the American Society for Clinical Oncology (ASCO), Chicago, IL, USA, June 2-6, 2017; and the 59th Annual Meeting of American Society for Therapeutic Radiology Oncology (ASTRO), San Diego, CA, USA, September 24-27, 2017.

\section{Disclosure}

The authors report no conflicts of interest in this work.

\section{References}

1. Vizcaino AP, Moreno V, Bosch FX, et al. International trends in incidence of cervical cancer. II: Squamous-cell carcinoma. Int J Cancer. 2000; 86(3):429-435.

2. Monk BJ, Tewari KS, Koh WJ. Multimodality therapy for locally advanced cervical carcinoma: state of the art and future directions. J Clin Oncol. 2007;25(20):2952-2965.

3. Thomas GM. Improved treatment for cervical cancer-concurrent chemotherapy and radiotherapy. $N$ Engl J Med. 1999;340(15):1198-1200.

4. Green JA, Kirwan JM, Tierney JF, et al. Survival and recurrence after concomitant chemotherapy and radiotherapy for cancer of the uterine cervix: a systematic review and meta-analysis. Lancet. 2001;358(9284): 781-786.

5. Neoadjuvant Chemotherapy for Locally Advanced Cervical Cancer Meta-analysis Collaboration. Neoadjuvant chemotherapy for locally advanced cervical cancer: a systematic review and meta-analysis of individual patient data from 21 randomised trials. Eur $J$ Cancer. 2003;39(17):2470-2486.

6. Chen H, Liang C, Zhang L, Huang S, Wu X. Clinical efficacy of modified preoperative neoadjuvant chemotherapy in the treatment of locally advanced (stage IB2 to IIB) cervical cancer: randomized study. Gynecol Oncol. 2008;110(3):308-315. 
7. Duenas-Gonzalez A, Lopez-Graniel C, Gonzalez-Enciso A, et al. Concomitant chemoradiation versus neoadjuvant chemotherapy in locally advanced cervical carcinoma: results from two consecutive phase II studies. Ann Oncol. 2002;13(8):1212-1219.

8. Modarress M, Maghami FQ, Golnavaz M, Behtash N, Mousavi A, Khalili GR. Comparative study of chemoradiation and neoadjuvant chemotherapy effects before radical hysterectomy in stage IB-IIB bulky cervical cancer and with tumor diameter greater than $4 \mathrm{~cm}$. Int $J$ Gynecol Cancer. 2005;15(3):483-488.

9. Ferrandina G, Gambacorta A, Gallotta V, et al. Chemoradiation with concomitant boosts followed by radical surgery in locally advanced cervical cancer: long-term results of the ROMA-2 prospective phase 2 study. Int J Radiat Oncol Biol Phys. 2014;90(4):778-784.

10. Ferrandina G, Ercoli A, Fagotti A, et al. Completion surgery after concomitant chemoradiation in locally advanced cervical cancer: a comprehensive analysis of pattern of postoperative complications. Ann Surg Oncol. 2014;21(5):1692-1699.

11. Wykosky J, Fenton T, Furnari F, Cavenee WK. Therapeutic targeting of epidermal growth factor receptor in human cancer: successes and limitations. Chin J Cancer. 2011;30(1):5-12.

12. Soonthornthum T, Arias-Pulido H, Joste N, et al. Epidermal growth factor receptor as biomarker for cervical cancer. Ann Oncol. 2011; 22(10):2166-2178.

13. Cetina L, Crombet T, Jiménez-Lima R, et al. A pilot study of nimotuzumab plus single agent chemotherapy as second- or third-line treatment or more in patients with recurrent, persistent or metastatic cervical cancer. Cancer Bio Ther. 2015;16(5):684-689.

14. Basavaraj C, Sierra P, Shivu J, Melarkode R, Montero E, Nair P. Nimotuzumab with chemoradiation confers a survival advantage in treatment-naïve head and neck tumors overpexpressing EGFR. Cancer Biol Ther. 2010;10(7):673-681.

15. McNeil C. New standard of care for cervical cancer sets stage for next questions. J Natl Cancer Inst. 1999;91(6):500-501.

16. Chemoradiotherapy for Cervical Cancer Meta-analysis Collaboration (CCCMAC). Reducing uncertainties about the effect of chemoradiotherapy for cervical cancer: individual patients data meta-analysis. Cochrane Database Syst Rev. 2010;1:CD008285.

17. Ferrandina G, Legge F, Fagotti A, et al. Preoperative concomitant chemoradiotherapy in locally advanced cervical cancer: safety, outcome, and prognostic measures. Gynecol Oncol. 2007;107(1 Suppl 1): S127-S132.

18. Koensgen D, Sehouli J, Belau A, et al. Clinical outcome of neoadjuvant radiochemotherapy in locally advanced cervical cancer. Int $J$ Gynecol Cancer. 2017;27(3):500-506.

19. Gong L, Zhang JW, Yin RT, et al. Safety and efficacy of neoadjuvant chemotherapy followed by radical surgery versus radical surgery alone in locally advanced cervical cancer patients. Int J Gynecol Cancer. 2016; 26(4):722-728
20. Lee KC, Moffat BA, Schott AF, et al. Prospective early response imaging biomarker for neoadjuvant breast cancer chemotherapy. Clin Cancer Res. 2007:13(2 Pt 1):443-450.

21. Pickles MD, Gibbs P, Lowry M, Turnbull LW. Diffusion changes precede size reduction in neoadjuvant treatment of breast cancer. Magn Reson Imaging. 2006;24(7):843-847.

22. Ferrandina G, Margariti PA, Smaniotto D, et al. Long-term analysis of clinical outcome and complication in locally advanced cervical cancer patients administered concomitant chemoradiation followed by radical surgery. Gyneco Oncol. 2010;119(3):404-410.

23. Macchia G, Cilla S, Deodato F, et al. Intensity-modulated extended-field chemoradiation plus simultaneous integrated boost in the pre-operative treatment of locally advanced cervical cancer: a dose-escalation study. Br J Radiol. 2015;88(1055):20150385.

24. Kato T, Nishimura H, Yakushiji M, et al. [Phase II study of 254-S (cis-diammine glycolato platinum) for gynecological cancer.] Gan to Kagaku Ryoho. 1992;19(5):695-701. Japanese.

25. Hartmann JT, Lipp HP. Toxicity of platinum compounds. Expert Opin Pharmacother. 2003;4(6):889-901.

26. Fujiwara M, Isohashi F, Mabuchi S, et al. Efficacy and safety of nedaplatin-based concurrent chemoradiotherapy for FIGO stage IB2-IVA cervical cancer and its clinical prognostic factors. J Radiat Res. 2015; 56(2):305-314

27. Vacchelli E, Aranda F, Eggermont A, et al. Trial watch: tumortargeting monoclonal antibodies in cancer therapy. Oncoimmunology. 2014;3(1):e27048.

28. Babu KG, Prabhash K, Vaid AK, et al. Nimotuzumab plus chemotherapy versus chemotherapy alone in advanced non-small-cell lung cancer: a multicenter, randomized, open-label phase II study. Onco Targets. 2014; 7:1051-1060.

29. Reddy BKM, Lokesh V, Vidyasagar MS, et al. Nimotuzumab provides survival benefit to patients with inoperable advanced squamous cell carcinoma of the head and neck: a randomized, open-label, phase IIb, 5-year study in India patients. Oral Oncol. 2014;50(5):498-505.

30. Garrido G, Tikhomirov IA, Rabasa A, et al. Bivalent binding by intermediate affinity of nimotuzumab: a contribution to explain antibody clinical profile. Cancer Biol Ther. 2011;11(4):373-382.

31. Movva S, Rodriguez L, Arias-Pulido H, Verschraegen C. Novel chemotherapy approaches for cervical cancer. Cancer. 2009;115(14): 3166-3180

32. Nicholson RI, Gee JM, Harper ME. EGFR and cancer prognosis. Eur J Cancer. 2001;37(Suppl 4):S9-S15.
OncoTargets and Therapy

\section{Publish your work in this journal}

OncoTargets and Therapy is an international, peer-reviewed, open access journal focusing on the pathological basis of all cancers, potential targets for therapy and treatment protocols employed to improve the management of cancer patients. The journal also focuses on the impact of management programs and new therapeutic agents and protocols on
Dovepress

patient perspectives such as quality of life, adherence and satisfaction The manuscript management system is completely online and includes a very quick and fair peer-review system, which is all easy to use. Visit http://www.dovepress.com/testimonials.php to read real quotes from published authors. 